Case Report

\title{
Migrating Polyarthritis as a Feature of Occult Malignancy: 2 Case Reports and a Review of the Literature
}

\author{
Geoffrey Alan Watson, ${ }^{1}$ Lorraine O'Neill, ${ }^{1}$ Ruth Law, ${ }^{1}$ \\ Geraldine McCarthy, ${ }^{2}$ and Douglas Veale ${ }^{1}$ \\ ${ }^{1}$ St. Vincent's University Hospital, Dublin, Ireland \\ ${ }^{2}$ Mater Misericordiae University Hospital, Dublin, Ireland \\ Correspondence should be addressed to Geoffrey Alan Watson; geoff_watson7@hotmail.com
}

Received 20 August 2015; Accepted 5 October 2015

Academic Editor: Annekathryn Goodman

Copyright (C) 2015 Geoffrey Alan Watson et al. This is an open access article distributed under the Creative Commons Attribution License, which permits unrestricted use, distribution, and reproduction in any medium, provided the original work is properly cited.

\begin{abstract}
Malignant disease may be associated with a wide variety of musculoskeletal syndromes. Rarely the musculoskeletal system can be indirectly affected by paraneoplastic phenomena, such as carcinomatous polyarthritis (CP). The differential diagnosis for CP is broad and is often a diagnosis of exclusion. CP often presents similarly to other forms of inflammatory arthritis, and a detailed history and physical examination can often distinguish CP from other more common causes of polyarticular arthritis. However serological tests such as rheumatoid factor (RF) and anti-citrullinated peptide (anti-CCP) antibody positivity, while rare, can be misleading. Clinical awareness and suspicion are paramount in achieving an accurate diagnosis and early detection of an occult neoplasm is critical for prompt management and therapy. We report two cases presenting with this unique clinical phenotype associated with paraneoplastic polyarthropathy and review the literature.
\end{abstract}

\section{Introduction}

Malignant disease may be associated with a wide variety of musculoskeletal syndromes [1-3]. Mechanisms include direct local invasion of the primary tumor, metastasis, and synovial response to juxta-articular masses. Rarely the musculoskeletal system can in addition be indirectly affected by paraneoplastic phenomena, such as carcinomatous polyarthritis (CP). CP is a poorly understood clinical entity and may present similarly to other forms of inflammatory arthritis. Distinguishing features have been proposed and include late age and abrupt onset, asymmetric joint involvement, negative family history, and resolution of symptoms after diagnosis and treatment of the underlying malignancy [2]. However $\mathrm{CP}$ remains a diagnosis of exclusion and positive serological tests such as rheumatoid factor (RF) may be misleading. It has previously been postulated that the absence of anticitrullinated peptide (anti-CCP) antibodies may distinguish $\mathrm{CP}$ from other more common rheumatic conditions such as rheumatoid arthritis (RA) [2]. However, while rare, isolated case reports are emerging linking $\mathrm{CP}$ with anti-CCP positivity, suggesting that serology may not be wholly reliable in providing this distinction. Here we report two cases presenting with a polyarthropathy including one case where anti-CCP antibodies were detectable.

\section{Case Reports}

Our first case involves 80-year-old female who presented with a three-day history of right-sided shoulder pain and associated fever. On further questioning the patient described asymmetric migratory joint pain over the preceding threeweek period, affecting her left wrist, knees, and right shoulder. She had been taking nonsteroidal anti-inflammatory drugs (NSAIDs) without relief and was also receiving a two-week course of ciprofloxacin for a urinary tract infection. Physical examination revealed tenderness and painful restriction of motion of the right shoulder. There was also a right-sided knee effusion. The remainder of the physical examination was unremarkable.

Her medical history was significant for type 2 diabetes, ischaemic heart disease, and congestive heart failure. She 


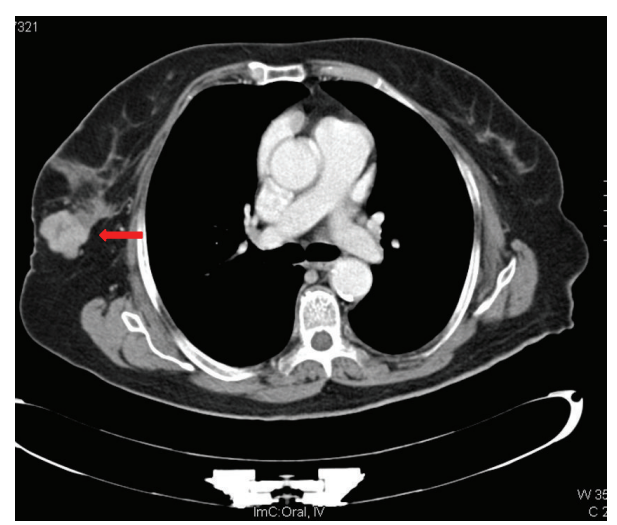

FIgURE 1: CT of abdomen. The red arrow illustrates a well circumscribed, multilobulated $3.3 \mathrm{~cm}$ lesion in the inferior outer quadrant of the right breast.

was a lifelong nonsmoker and did not consume any alcohol. Review of systems was otherwise unremarkable.

Initial laboratory results revealed a white cell count of $11.9 \times 10^{9} / \mathrm{L}$, hemoglobin $11.2 \mathrm{~g} / \mathrm{dL}$, and platelets $384 \times 10^{9} / \mathrm{L}$. A metabolic panel was significant for mild hyponatremia (131 mmol/L) (normal levels $135-145 \mathrm{mmol} / \mathrm{L}$ ) and a slightly raised urea $(7.7 \mathrm{mmol} / \mathrm{L})$ and creatinine $(93 \mu \mathrm{mol} / \mathrm{L})$. Her erythrocyte sedimentation rate (ESR) was $21 \mathrm{~mm} / \mathrm{h}$ and Creactive protein (CRP) was elevated at $111.8 \mathrm{mg} / \mathrm{L}$. Uric acid was $0.29 \mathrm{mmol} / \mathrm{L}$. Rheumatoid factor was marginally elevated at $42 \mathrm{IU} / \mathrm{mL}$. Anti-CCP was also elevated at $36 \mathrm{IU} / \mathrm{mL}$. An $\mathrm{X}$-ray of her right shoulder showed moderate degenerative changes at the acromioclavicular joint. Imaging of the knees and pelvis showed mild degenerative changes. Urine dipstick was positive for leukocytes but negative for nitrites. She was initially suspected of having polyarticular gout based on her slightly elevated uric acid levels and was subsequently treated with colchicine.

During her admission she developed nonspecific abdominal pain and tenderness in the right iliac fossa. A CT of abdomen was requested and reported no intra-abdominal pathology. However there was an incidental finding of a $3.3 \mathrm{~cm}$ multilobulated lesion in the inferior outer quadrant of the right breast (Figure 1). Subsequent mammogram revealed a $3.5 \mathrm{~cm}$ irregular hypoechoic mass highly suggestive of malignancy. This was confirmed after tissue biopsy, with microscopy revealing a papillary carcinoma (Figure 2).

During that time her right shoulder pain spontaneously resolved. However she then developed new onset right wrist pain, swelling, and erythema. X-ray revealed moderate degenerative changes. Joint aspiration revealed markedly increased inflammatory cells but no crystals. Colchicine and NSAIDs were stopped and she was given IM methylprednisolone to which she responded well, and her CRP decreased to 70 . She was seen in clinic one week later and had no appreciable synovitis on examination.

Six weeks later she underwent chemotherapy and radiotherapy for her breast cancer. She was seen again in clinic ten weeks later and she did not report any recurrence of her joint symptoms.

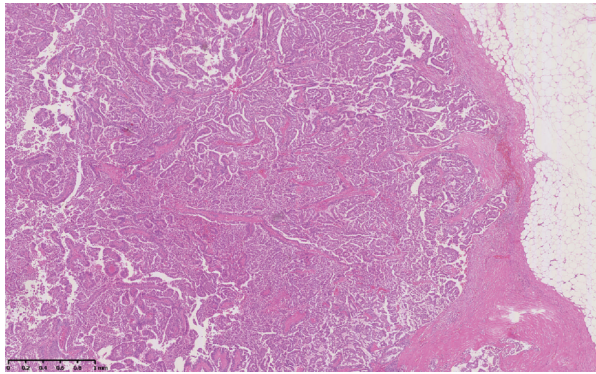

FIGURE 2: Encapsulated papillary carcinoma showing classical papillary architecture with surrounding fibrous capsule.

Our second patient, 71-year-old female, was referred by her general practitioner for evaluation of a six-month history of pain and swelling of her hands and feet. This was associated with early morning stiffness lasting greater than two hours daily. NSAIDs and a short course of prednisolone had failed to improve her symptoms.

Her past medical history was significant for ischaemic heart disease, hypertension, hyperlipidaemia, and peptic ulcer disease. She had a thirty-pack year history of smoking. Three of her four siblings had been treated for colorectal or breast neoplasms.

On examination she had thirteen tender and ten swollen joints including metacarpophalangeal (MCP), proximal interphalangeal (PIP), wrist, shoulder, ankle, and knee joints. Grade 2 clubbing was evident as was a multinodular goitre. Other than a raised CRP at $12 \mathrm{mg} / \mathrm{L}$ and serum calcium at $2.68 \mathrm{mmol} / \mathrm{L}$, baseline laboratory investigations were normal. Rheumatoid factor and anti-CCP antibodies were both negative. Chest radiograph revealed a left upper lobe lesion, which was confirmed on CT. Subsequent biopsy identified a non-small cell adenocarcinoma. She underwent a lobectomy with a rapid resolution of her joint symptoms.

\section{Discussion}

Migratory polyarthritis is a common presentation with a broad differential diagnosis [1-4]. Common causes include inflammatory arthritis including crystal arthropathy and other forms of systemic rheumatic disease such as connective tissue disorders and occasionally infectious agents [4]. Less commonly, joint symptoms may be attributed to neoplastic disease.

$\mathrm{CP}$ is associated with a wide variety of solid tumours and haematologic malignancies, particularly cancers of the lung and ovaries (Table 1). Cancer polyarthritis was first described by Lansbury in 1953 [5] and by MacKenzie and Scherbel in 1963 [6]. The topic has since been reviewed by Naschitz et al. [7] and most recently by Larson et al. [8]. Hypertrophic osteoarthropathy, dermatomyositis, paraneoplastic vasculitis, and most recently palmar fasciitis have all been described as paraneoplastic disorders [1,9]. However, carcinomatous polyarthritis remains a rare and poorly understood clinical entity $[2,3]$. CP is historically defined by the following criteria $[1,2]$ : 
TABLE 1: Malignancies associated with carcinomatous polyarthritis. Four cases, highlighted in bold, have shown anti-CCP positivity. Rheumatoid factor (RF), anti-citrullinated protein antibody (anti-CCP), positive (+), negative (-), not included (NI), Non-Small Cell Lung Cancer (NSCLC), Squamous Cell Cancer (SCC), Small Cell Lung Cancer (SCLC), Acute Lymphoblastic Leukemia (ALL), Chronic Myeloid Leukemia (CML), and Chronic Lymphoid Leukemia (CLL).

\begin{tabular}{|c|c|c|c|}
\hline Case report and reference & Type of malignancy & $\mathrm{RF}$ & Anti-CCP \\
\hline Present case & Breast & + & + \\
\hline Present case & NSCLC & - & - \\
\hline Zupancic et al. [2] & SCC lung & + & $\mathrm{NI}$ \\
\hline Stummvoll et al. [3] & Adenocarcinoma colon & - & NI \\
\hline Stummvoll et al. [3] & SCC Lung & - & NI \\
\hline Larson et al. [8] & NSCLC (adenocarcinoma) & + & + \\
\hline Nadal et al. [10] & Prostate cancer & NI & - \\
\hline Bradley and Pinals [11] & Spindle cell cancer & + & $\mathrm{NI}$ \\
\hline Pines et al. [12] & Breast & - & NI \\
\hline Pines et al. [12] & Unknown primary & - & NI \\
\hline Pines et al. [12] & SCLC & - & NI \\
\hline Acosta Madiedo et al. [13] & NSCLC & - & $\mathrm{NI}$ \\
\hline Chuan et al. [9] & Tubular adenocarcinoma stomach & - & $\mathrm{NI}$ \\
\hline Eggelmeijer and Macfarlane [14] & Supraglottic SCC & - & $\mathrm{NI}$ \\
\hline Bennett et al. [15] & Ovarian adenocarcinoma & + & $\mathrm{NI}$ \\
\hline Simon and Ford [16] & Adenocarcinoma colon & - & $\mathrm{NI}$ \\
\hline Mok and Kwan [17] & Unknown primary & + & $\mathrm{NI}$ \\
\hline Handy et al. [18] & T cell ALL & + & + \\
\hline Kumar et al. [19] & Pancreas & + & + \\
\hline Sheehy et al. [20] & NSCLC (adenocarcinoma) & + & NI \\
\hline Bivalacqua et al. [21] & NSCLC (adenocarcinoma) & NI & $\mathrm{NI}$ \\
\hline Docquier et al. [22] & Uterine adenocarcinoma & - & $\mathrm{NI}$ \\
\hline Haroon and Phelan [23] & Pancreatic cancer & - & $\mathrm{NI}$ \\
\hline Ardalan and Shoja [24] & Multiple myeloma & - & $\mathrm{NI}$ \\
\hline Leslie [25] & Cervical cancer & NI & NI \\
\hline Baijens and Manni [26] & cT4N2cM0 hypopharynx carcinoma & NI & NI \\
\hline Martorell et al. [27] & Serocystadenocarcinoma ovary & - & $\mathrm{NI}$ \\
\hline Martorell et al. [27] & Serocystadenocarcinoma ovary & + & NI \\
\hline Martorell et al. [27] & Ovarian carcinoma & - & $\mathrm{NI}$ \\
\hline Martorell et al. [27] & Serocystadenocarcinoma ovary & - & NI \\
\hline Medsger et al. [28] & Ovarian adenocarcinoma & NI & $\mathrm{NI}$ \\
\hline Medsger et al. [28] & Ovarian adenocarcinoma & NI & NI \\
\hline Medsger et al. [28] & Ovarian adenocarcinoma & NI & NI \\
\hline Medsger et al. [28] & Ovarian adenocarcinoma & NI & NI \\
\hline Medsger et al. [28] & Ovarian adenocarcinoma & NI & NI \\
\hline Medsger et al. [28] & Ovarian adenocarcinoma & NI & $\mathrm{NI}$ \\
\hline Baron [29] & SCLC & - & NI \\
\hline Baer and Phillips [30] & Pancreatic adenocarcinoma & NI & NI \\
\hline Taggart et al. [31] & Adenocarcinoma Fallopian tube & - & NI \\
\hline Taggart et al. [31] & Ovarian adenocarcinoma & NI & NI \\
\hline Michaels and Sorber [32] & Pancreatic adenocarcinoma & - & NI \\
\hline Shiel et al. [33] & SCLC & NI & $\mathrm{NI}$ \\
\hline Shiel et al. [33] & Ovarian adenocarcinoma & NI & NI \\
\hline Pfinsgraff et al. [34] & CML & - & NI \\
\hline Pfinsgraff et al. [34] & Pancreatic adenocarcinoma, parathyroid adenoma & NI & NI \\
\hline Pfinsgraff et al. [34] & Squamous cell carcinoma unknown primary & - & NI \\
\hline Pfinsgraff et al. [34] & Adenocarcinoma unknown primary & NI & $\mathrm{NI}$ \\
\hline
\end{tabular}


TABLE 1: Continued.

\begin{tabular}{|c|c|c|c|}
\hline Case report and reference & Type of malignancy & $\mathrm{RF}$ & Anti-CCP \\
\hline Pfinsgraff et al. [34] & Hodgkin disease & NI & NI \\
\hline Valverde-Garcia et al. [35] & Breast cancer & NI & $\mathrm{NI}$ \\
\hline Cammilleri et al. [36] & Follicular B cell lymphoma & - & NI \\
\hline Willemse et al. [37] & Adenocarcinoma of the coelomic epithelium & - & $\mathrm{NI}$ \\
\hline Van den Bergh et al. [38] & Adenocarcinoma of prostate prolactinoma & - & $\mathrm{NI}$ \\
\hline Mathieu et al. [39] & CLL & NI & $\mathrm{NI}$ \\
\hline Vinker et al. [40] & Ovarian adenocarcinoma & NI & $\mathrm{NI}$ \\
\hline Saxman and Seitz [41] & Breast carcinoma & - & $\mathrm{NI}$ \\
\hline Grados et al. [42] & Transitional cell carcinoma of the renal pelvis & - & $\mathrm{NI}$ \\
\hline Grados et al. [42] & Adenocarcinoma of the uterus & - & NI \\
\hline Enomoto et al. [43] & Early-stage gastric carcinoma & + & $\mathrm{NI}$ \\
\hline Denschlag et al. [44] & Fallopian tube carcinoma & - & NI \\
\hline Giannakopoulos et al. [45] & Ovarian adenocarcinoma & - & NI \\
\hline Yogarajah et al. [46] & Ovarian adenocarcinoma & NI & $\mathrm{NI}$ \\
\hline Bolibar et al. [47] & Ovarian adenocarcinoma & - & NI \\
\hline Preda et al. [48] & Ovarian adenocarcinoma & NI & NI \\
\hline Krishna et al. [49] & Breast cancer & - & NI \\
\hline Qureshi and Saavedra [50] & Ovarian adenocarcinoma & - & NI \\
\hline Clarke et al. [51] & Transitional cell carcinoma of the bladder & - & NI \\
\hline Nahar and Al-Rajhi [52] & Ovarian adenocarcinoma & - & NI \\
\hline Sandhya and Danda [53] & Breast carcinoma & NI & NI \\
\hline Mcgivern and Mcaleese [54] & NSCLC & NI & $\mathrm{NI}$ \\
\hline Salmon et al. [55] & Ovarian adenocarcinoma & - & NI \\
\hline Salmon et al. [55] & Ovarian adenocarcinoma & - & NI \\
\hline Salmon et al. [55] & Uterine cancer & NI & NI \\
\hline Shetty et al. [56] & Neuroendocrine tumour of adrenal gland & - & $\mathrm{NI}$ \\
\hline
\end{tabular}

(i) Close temporal relationship (12 months) between onset of arthritis and malignancy

(ii) Late age at onset

(iii) Asymmetric joint involvement

(iv) Explosive onset

(v) Predominance of lower extremity involvement with sparing of wrists and small joints of hands

(vi) Absence of Rheumatoid Factor (RF)

(vii) Absence of rheumatoid nodules

(viii) No family history of rheumatic disease

(ix) Non-specific histopathologic appearance of synovial lining

(x) Absence of characterisitic radiologic lesions

Box 1: Features of cancer polyarthritis (Stummvoll et al. [3], Larson et al. [8], Caldwell and McCallum [57], and Pfitzenmeyer et al. [58]).

(i) It must occur during the course of an identified malignant disease or precede clinical evidence of a malignancy.

(ii) Symptoms cannot be the result of direct tumor invasion or compression.

(iii) Symptoms improve with treatment of the underlining neoplasm.

In 1985, Caldwell and McCallum published the key features of cancer polyarthritis and suggested distinguishing features to aid in narrowing the differential [57]. Pfitzenmeyer et al. [58] later added the absence of characteristic radiologic lesions to the list of typical features (Box 1). These features have been supported by recent case reviews $[2,3,8,10]$. It is now well recognised that rheumatic syndromes often precede the diagnosis of malignant disease $[1-3,8,57]$. Racanelli et al. found that, in patients with solid tumours, only $11.5 \%$ had a malignancy diagnosed at the time of their rheumatic presentation, while $88.5 \%$ were diagnosed after their initial presentation [59]. Thus new presentations of polyarthropathy may represent an important clue in the detection of an occult malignancy.

CP however remains a diagnosis of exclusion and must be distinguished from other more common causes of 
polyarthritis. Infectious causes due to bacteria or Lyme disease may be associated with fever, acute symptom onset, and monoarthritis. A thorough clinical history is useful in detecting seronegative spondyloarthropathies. Reactive arthritis is usually preceded by an infection such as campylobacterinduced diarrhea, while enteric arthritis may be seen in patients with a history of inflammatory bowel disease. Finally the crystal arthropathies can be easily distinguished by joint aspiration and visualisation of crystals under the microscope.

Distinguishing CP from a late onset form of RA, however, is challenging. Previous studies have shown that the incidence of both increases with age. Both may present over the course of weeks to months with similar signs and symptoms, namely, soft tissue swelling, limited range of motion of affected joints, and morning stiffness [3, 9, 11-16]. Features suggestive of chronic disease and inflammation (anaemia, elevated ESR/CRP) are also seen $[2,3,11-13,16,20,22,28$, $29,42,49,55]$. The joint fluid generally shows nonspecific inflammatory changes $[1,2,11,16,17]$. Imaging studies are largely unremarkable $[1-3,14,16,60]$. In addition symptoms often fail to resolve with the use of conventional therapy such as NSAIDs and steroids [2, 27-32, 41, 42, 44, 48, 50].

Several case reports included in Box 1 have emerged documenting findings that challenge the traditional distinguishing features of CP. Several noted symmetric joint involvement involving the wrists, knees, and small joints of the hands as the predominant rheumatic manifestation $[2,3,8-16,23,27,28,31,34,35,38-40,42,43,48,49]$.

Our first case had a positive rheumatoid factor, which can be partially explained by the underlying malignancy, which is associated with a positive rheumatoid factor in 10-20 percent of patients [58]. Morel et al. documented symptom relief for 9 out of 20 patients who were treated with NSAIDs, while most responded to steroid therapy [60].

An additional means to differentiate these two disorders was postulated by measuring anti-cyclic citrullinated peptide (anti CCP) antibodies. Anti-CCP has a similar sensitivity to RF (50-75\%) with a higher specificity (90-95\%) [61]. Due to this high specificity, however, a positive Anti-CCP may result in an inaccurate diagnosis of rheumatoid arthritis, thus resulting in delayed diagnosis and treatment of the underlying malignancy. To our knowledge, only four case reports, including the present case, have reported an association between CP and elevated anti-CCP levels [8, 18, 19] (Box 1). Thus, many features that have been proposed to distinguish between CP and RA are not always reliable.

One prevailing theme however is resolution of rheumatic symptoms after treatment of the underlying malignancy $[2,3$, $9,11-16,22,27,28,33-35,39-41,43-48,50,52,55]$. Resolution of symptoms after chemotherapy or surgery may vary, and while our first patient's symptoms may have been masked by steroids, our second patient showed a rapid improvement just days after undergoing surgery. The return of arthritic symptoms in these patients should provoke further investigation to rule out any evidence of tumour recurrence [2].

To date, the pathogenesis of this disorder remains unclear. Bradley and Pinals suggested that circulating immune complexes (CIC), which have been observed in over 60 percent of some types of cancers, may play a pivotal role [11].
Theories suggesting that elevated levels of platelet activating factors may be deposited in the synovium and trigger an inflammatory response have also been postulated [2, 15]. Additional mechanisms have been proposed including autoimmune phenomena involving lymphocytes originating in hyperplastic lymph nodes draining tumour sites $[1,9,11$, 14]; however these theories all remain speculative.

\section{Summary}

Carcinomatous polyarthritis is a rare disorder associated with a wide variety of malignancies. The differential diagnosis for $\mathrm{CP}$ is broad and is often a diagnosis of exclusion. Several of the classical features initially proposed to distinguish CP may be unreliable, and positive serological tests such as rheumatoid factor and anti-CCP antibody positivity can be misleading. A comprehensive history and physical examination is paramount in distinguishing CP from other more common causes of polyarticular arthritis, with careful attention to social and family history to detect any possible risk factors for cancer. Clinical awareness and suspicion remain the most important factors in achieving an accurate diagnosis. Early diagnosis of an occult neoplasm is critical for prompt management and therapy and can ultimately be lifesaving.

\section{Conflict of Interests}

The authors declare that there is no conflict of interests regarding the publication of this paper.

\section{References}

[1] G. Fam Adel, "Paraneoplastic rheumatic syndromes," Baillière’s Best Practice \& Research. Clinical Rheumatology, vol. 14, no. 3, pp. 515-533, 2000.

[2] M. Zupancic, A. Annamalai, J. Brenneman, and S. Ranatunga, "Migratory polyarthritis as a paraneoplastic syndrome," Journal of General Internal Medicine, vol. 23, no. 12, pp. 2136-2139, 2008.

[3] G. H. Stummvoll, M. Aringer, K. P. Machold, J. S. Smolen, and M. Raderer, "Cancer polyarthritis resembling rheumatoid arthritis as a first sign of hidden neoplasms," Scandinavian Journal of Rheumatology, vol. 30, no. 1, pp. 40-44, 2001.

[4] A. M. Richie and M. L. Francis, "Diagnostic approach to polyarticular joint pain," American Family Physician, vol. 68, no. 6, pp. 1151-1160, 2003.

[5] J. Lansbury, "Collagen disease complicating malignancy," Annals of the Rheumatic Diseases, vol. 12, no. 4, pp. 301-305, 1953.

[6] A. H. MacKenzie and A. L. Scherbel, "Connective tissue syndromes associated with carcinoma," Geriatrics, vol. 18, article 753, 1963.

[7] J. E. Naschitz, I. Rosner, M. Rozenbaum, E. Zuckerman, and D. Yeshurun, "Rheumatic syndromes: clues to occult neoplasia," Seminars in Arthritis and Rheumatism, vol. 29, no. 1, pp. 43-55, 1999.

[8] E. Larson, D. Etwaru, C. Siva, and K. Lawlor, "Report of antiCCP antibody positive paraneoplastic polyarthritis and review of the literature," Rheumatology International, vol. 31, no. 12, pp. 1635-1638, 2011. 
[9] H. Chuan, C. E. Robinson, W. H. Sutherland, and W. L. Dunn, "Polyarthritis associated with gastric carcinoma," Canadian Medical Association Journal, vol. 131, no. 8, pp. 909-911, 1984.

[10] R. Nadal, Z. H. McMahan, and E. S. Antonarakis, "Paraneoplastic palmar fasciitis and polyarthritis syndrome in a patient with advanced prostate cancer," Clinical Genitourinary Cancer, vol. 11, no. 4, pp. e15-e23, 2013.

[11] J. D. Bradley and R. S. Pinals, "Carcinoma polyarthritis: role of immune complexes in pathogenesis," Journal of Rheumatology, vol. 10, no. 5, pp. 826-828, 1983.

[12] A. Pines, N. Kaplinsky, D. Olchovsky, and O. Frankl, "Rheumatoid arthritis-like syndrome: a presenting symptom of malignancy. Report of 3 cases and review of the literature," European Journal of Rheumatology and Inflammation, vol. 7, no. 2, pp. 51$55,1984$.

[13] J. M. Acosta Madiedo, A. Murthy, D. A. Cortese, and M. D. Cohen, "An unusual case of carcinoma polyarthritis with associated vasculitis," Arthritis and Rheumatism, vol. 40, no. 4, pp. 779-782, 1997.

[14] F. Eggelmeijer and J. D. Macfarlane, "Polyarthritis as the presenting symptom of the occurrence and recurrence of a laryngeal carcinoma," Annals of the Rheumatic Diseases, vol. 51, no. 4, pp. 556-557, 1992.

[15] R. M. Bennett, M. H. Ginsberg, and S. Thomsen, "Carcinomatous polyarthritis: the presenting symptom of an ovarian tumor and association with a platelet activating factor," Arthritis \& Rheumatism, vol. 19, no. 5, pp. 953-958, 1976.

[16] R. D. Simon Jr. and L. E. Ford, "Rheumatoid-like arthritis associated with a colonic carcinoma," Archives of Internal Medicine, vol. 140, no. 5, pp. 698-700, 1980.

[17] C. C. Mok and Y. K. Kwan, "Rheumatoid-like polyarthritis as a presenting feature of metastatic carcinoma: a case presentation and review of the literature," Clinical Rheumatology, vol. 22, no. 4-5, pp. 353-354, 2003.

[18] C. E. Handy, G. Robles, U. Haque, and B. Houston, “T cell ALL presenting as seropositive rheumatoid arthritis: case report and review of the literature on seropositive paraneoplastic arthritis," Clinical Rheumatology, vol. 34, no. 9, pp. 1647-1650, 2015.

[19] S. Kumar, S. Sethi, F. Irani, and B. Y. Bode, "Anticyclic citrullinated peptide antibody-positive paraneoplastic polyarthritis in a patient with metastatic pancreatic cancer," The American Journal of the Medical Sciences, vol. 338, no. 6, pp. 511-512, 2009.

[20] C. Sheehy, J. G. Ryan, M. Kelly, and M. Barry, "Palmar fasciitis and polyarthritis syndrome associated with non-small-cell lung carcinoma," Clinical Rheumatology, vol. 26, no. 11, pp. 1951-1953, 2007.

[21] T. J. Bivalacqua, H. Alphs, I. Aksentijevich, E. M. Schaeffer, and M. P. Schoenberg, "Paraneoplastic polyarthritis from nonsmall-cell lung cancer metastatic to the bladder," Journal of Clinical Oncology, vol. 25, no. 18, pp. 2621-2623, 2007.

[22] C. Docquier, F. Majois, and C. Mitine, "Palmar fasciitis and arthritis: association with endometrial adenocarcinoma," Clinical Rheumatology, vol. 21, no. 1, pp. 63-65, 2002.

[23] M. Haroon and M. Phelan, "A paraneoplastic case of palmar fasciitis and polyarthritis syndrome," Nature Clinical Practice Rheumatology, vol. 4, no. 5, pp. 274-277, 2008.

[24] M. R. Ardalan and M. M. Shoja, "Multiple myeloma presented as acute interstitial nephritis and rheumatoid arthritis-like polyarthritis," American Journal of Hematology, vol. 82, no. 4, pp. 309-313, 2007.
[25] B. M. Leslie, "Palmar fasciitis and polyarthritis associated with a malignant neoplasm: a paraneoplastic syndrome," Orthopedics, vol. 15, no. 12, pp. 1436-1439, 1992.

[26] L. W. J. Baijens and J. J. Manni, "Paraneoplastic syndromes in patients with primary malignancies of the head and neck. Four cases and a review of the literature," European Archives of OtoRhino-Laryngology, vol. 263, no. 1, pp. 32-36, 2006.

[27] E. A. Martorell, P. M. Murray, J. J. Peterson, D. M. Menke, and K. T. Calamia, "Palmar fasciitis and arthritis syndrome associated with metastatic ovarian carcinoma: a report of four cases," Journal of Hand Surgery, vol. 29, no. 4, pp. 654-660, 2004.

[28] T. A. Medsger Jr., J. A. Dixon, and V. F. Garwood, "Palmar fasciitis and polyarthritis associated with ovarian carcinoma," Annals of Internal Medicine, vol. 96, no. 4, pp. 424-431, 1982.

[29] M. Baron, "Palmar fasciitis, polyarthritis, and carcinoma," Annals of Internal Medicine, vol. 97, article 616, 1982.

[30] A. N. Baer and R. M. Phillips Jr., "Pancreatic carcinoma and palmar fasciitis," Annals of Internal Medicine, vol. 99, no. 3, pp. 411-412, 1983.

[31] A. J. Taggart, J. M. I. Iveson, and V. Wright, "Shoulder-hand syndrome and symmetrical arthralgia in patients with tuboovarian carcinoma," Annals of the Rheumatic Diseases, vol. 43, no. 3, pp. 391-393, 1984.

[32] R. M. Michaels and J. A. Sorber, "Reflex sympathetic dystrophy as a probable paraneoplastic syndrome: case report and literature review," Arthritis and Rheumatism, vol. 27, no. 10, pp. 1183$1185,1984$.

[33] W. C. Shiel Jr., P. E. Prete, M. Jason, and B. S. Andrews, "Palmar fasciitis and arthritis with ovarian and non-ovarian carcinomas. New syndrome," The American Journal of Medicine, vol. 79, no. 5, pp. 640-644, 1985.

[34] J. Pfinsgraff, R. B. Buckingham, P. J. Killian et al., "Palmar fasciitis and arthritis with malignant neoplasms: a paraneoplastic syndrome," Seminars in Arthritis and Rheumatism, vol. 16, no. 2, pp. 118-125, 1986.

[35] J. Valverde-Garcia, X. Juanola-Roura, J. M. Ruiz-Martin, J. M. Nolla-Sole, J. Rodriguez-Moreno, and D. Roig-Escofet, "Paraneoplastic palmar fasciitis-polyarthritis syndrome associated with breast cancer," Journal of Rheumatology, vol. 14, no. 6, pp. 12071209, 1987.

[36] S. Cammilleri, M. C. Guzian, J. Mattei, O. Mundler, and S. Guis, "Whole-body ${ }^{18}$ FDG-PET in an arthritis paraneoplastic syndrome revealed an underlying hematological neoplasm," Case Reports in Medicine, vol. 2013, Article ID 594704, 3 pages, 2013.

[37] P. H. B. Willemse, N. H. Mulder, H. J. van de Tempel, J. G. Aalders, and D. T. Sleijfer, "Palmar fasciitis and arthritis in a patient with an extraovarian adenocarcinoma of the coelomic epithelium," Annals of the Rheumatic Diseases, vol. 50, no. 1, pp. 53-54, 1991.

[38] L. Van den Bergh, S. B. Vanneste, and D. C. Knockaert, "Palmar fasciitis and arthritis associated with cancer of the prostate.", Acta Clinica Belgica, vol. 46, no. 2, pp. 106-110, 1991.

[39] E. Mathieu, A. C. Koeger, A. Coutellier, S. Rozenberg, B. De La Tour, and P. Bourgeois, "Palmar fasciitis arthritis syndrome revealing relapse of chronic lymphoid leukemia," Presse Medicale, vol. 20, no. 11, article 521, 1991.

[40] S. Vinker, R. Dgani, B. Lifschitz-Mercer, Z. M. Sthoeger, and L. Green, "Palmar fasciitis and polyarthritis associated with ovarian carcinoma in a young patient: a case report and review of the literature," Clinical Rheumatology, vol. 15, no. 5, pp. 495497, 1996. 
[41] S. B. Saxman and D. Seitz, "Breast cancer associated with palmar fasciitis and arthritis," Journal of Clinical Oncology, vol. 15, no. 12, pp. 3515-3516, 1997.

[42] F. Grados, E. Houvenagel, G. Cayrolle, R. Bellony, P. Fardellone, and L. Sebert J.-, "Two new cancer locations accompanied with palmar fasciitis and polyarthritis," Revue du Rhumatisme (English Edition), vol. 65, no. 3, pp. 212-214, 1998.

[43] M. Enomoto, H. Takemura, M. Suzuki et al., "Palmar fasciitis and polyarthritis associated with gastric carcinoma: complete resolution after total gastrectomy," Internal Medicine, vol. 39, no. 9, pp. 754-757, 2000.

[44] D. Denschlag, E. Riener, P. Vaith, C. Tempfer, and C. Keck, "Palmar fasciitis and polyarthritis as a paraneoplastic syndrome associated with tubal carcinoma: a case report," Annals of the Rheumatic Diseases, vol. 63, no. 9, pp. 1177-1178, 2004.

[45] C. K. Giannakopoulos, G. K. Kyriakidou, and G. E. Toufexi, "Palmar fasciitis and polyarthritis associated with secondary ovarian carcinoma. Case report," European Journal of Gynaecological Oncology, vol. 26, no. 3, pp. 339-341, 2005.

[46] M. Yogarajah, J. Soh, B. Lord, N. Goddard, and R. Stratton, "Palmar fasciitis and polyarthritis syndrome: a sign of ovarian malignancy," Journal of the Royal Society of Medicine, vol. 101, no. 9, pp. 473-475, 2008.

[47] E. P. Bolibar, J. S. Raya, C. P. Simeon, L. Montesinos, N. Jou, and A. Cuxart, "Palmar fascitis and polyarthritis as a paraneoplastic syndrome associated with ovarian carcinoma: a case report," BMJ Case Reports, vol. 2009, 2009.

[48] V. A. Preda, P. Frederiksen, and S. Kossard, "Indurated reticulate palmar erythema as a sign of paraneoplastic palmar fasciitis and polyarthritis syndrome," Australasian Journal of Dermatology, vol. 50, no. 3, pp. 198-201, 2009.

[49] K. Krishna, A. Yacoub, L. F. Hutchins, and P. M. Kortebein, "Palmar fasciitis with polyarthritis syndrome in a patient with breast cancer," Clinical Rheumatology, vol. 30, no. 4, pp. 569572, 2011

[50] A. A. Qureshi and A. Saavedra, "Palmar fasciitis and polyarthritis syndrome in patients with ovarian cancer-a case report and review of the literature," Hand, vol. 6, no. 2, pp. 220-223, 2011.

[51] L. L. Clarke, C. T. C. Kennedy, and P. Hollingworth, "Palmar fasciitis and polyarthritis syndrome associated with transitional cell carcinoma of the bladder," Journal of the American Academy of Dermatology, vol. 64, no. 6, pp. 1159-1163, 2011.

[52] I. K. Nahar and M. S. Al-Rajhi, "Palmar fasciitis and arthritis syndrome associated with metastatic ovarian cancer: a paraneoplastic syndrome," The Gulf Journal of Oncology, no. 12, pp. 5961, 2012.

[53] P. Sandhya and D. Danda, "Paraneoplastic palmar fasciitis in carcinoma breast," Journal of Clinical Rheumatology, vol. 18, no. 2, article 112, 2012.

[54] U. Mcgivern and J. Mcaleese, "Palmar fasciitis: a para-neoplastic phenomenon indicating recurrence of non small cell lung cancer-case report and review of the literature," International Journal of Rheumatic Diseases, vol. 15, no. 1, pp. 8-9, 2012.

[55] C. Salmon, G. Gill, D. Loeuille, and I. Chary-Valckenaere, "Three new cases of palmar fasciitis with polyarthritis including a patient with atypical mutilating osteolysis," Joint Bone Spine, vol. 80, no. 2, pp. 217-220, 2013.

[56] S. Shetty, J. Hephzibah, B. Borah et al., "Paraneoplastic polyarthritis in association with metastatic neuroendocrince tumour of the adrenal gland," The Australasian Medical Journal, vol. 7, no. 8, pp. 345-349, 2014.
[57] D. S. Caldwell and R. M. McCallum, "Rheumatologic manifestations of cancer," Medical Clinics of North America, vol. 70, no. 2, pp. 385-417, 1986.

[58] P. Pfitzenmeyer, P. Bielefeld, C. Tavernier, J. F. Besancenot, and M. Gaudet, "Current aspects of paraneoplastic acute polyarthritis," La Revue de Medecine Interne, vol. 13, no. 3, pp. 195-199, 1992.

[59] V. Racanelli, M. Prete, C. Minoia, E. Favoino, and F. Perosa, "Rheumatic disorders as paraneoplastic syndromes," Autoimmunity Reviews, vol. 7, no. 5, pp. 352-358, 2008.

[60] J. Morel, V. Deschamps, E. Toussirot et al., "Characteristics and survival of 26 patients with paraneoplastic arthritis," Annals of the Rheumatic Diseases, vol. 67, no. 2, pp. 244-247, 2008.

[61] A. Wilk and W.van Venrooij, The Use of Anti-Cyclic Citrullinated Peptide (anti-CCP) Antibodies in RA, American College of Rheumatology, 2003. 


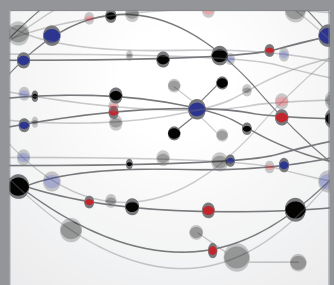

The Scientific World Journal
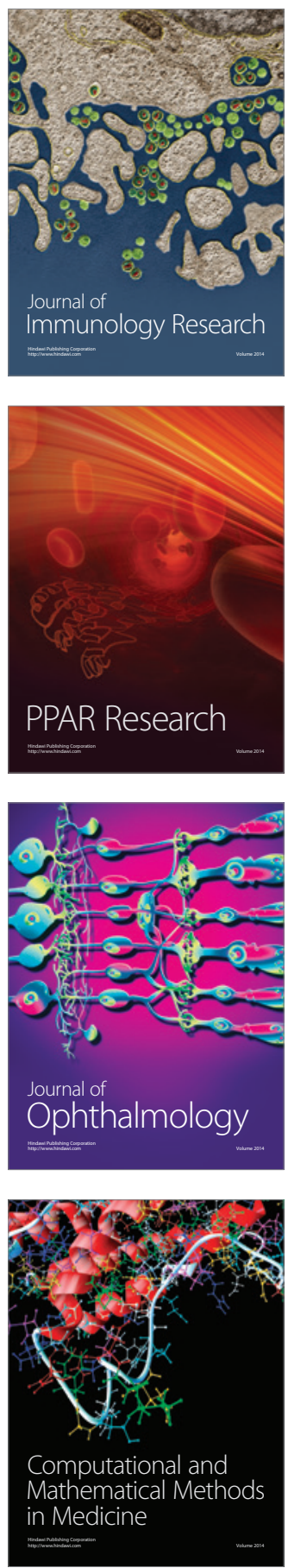

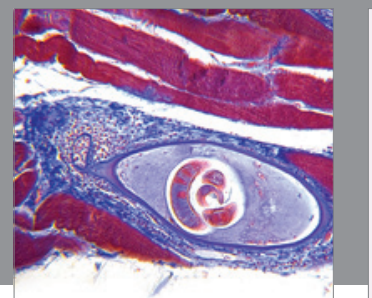

Gastroenterology

Research and Practice
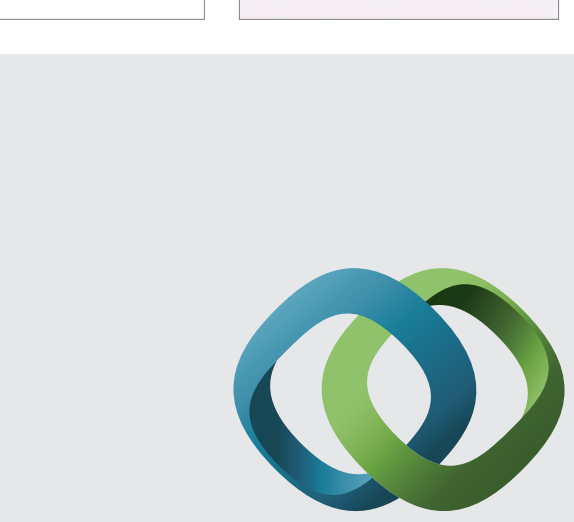

\section{Hindawi}

Submit your manuscripts at

http://www.hindawi.com
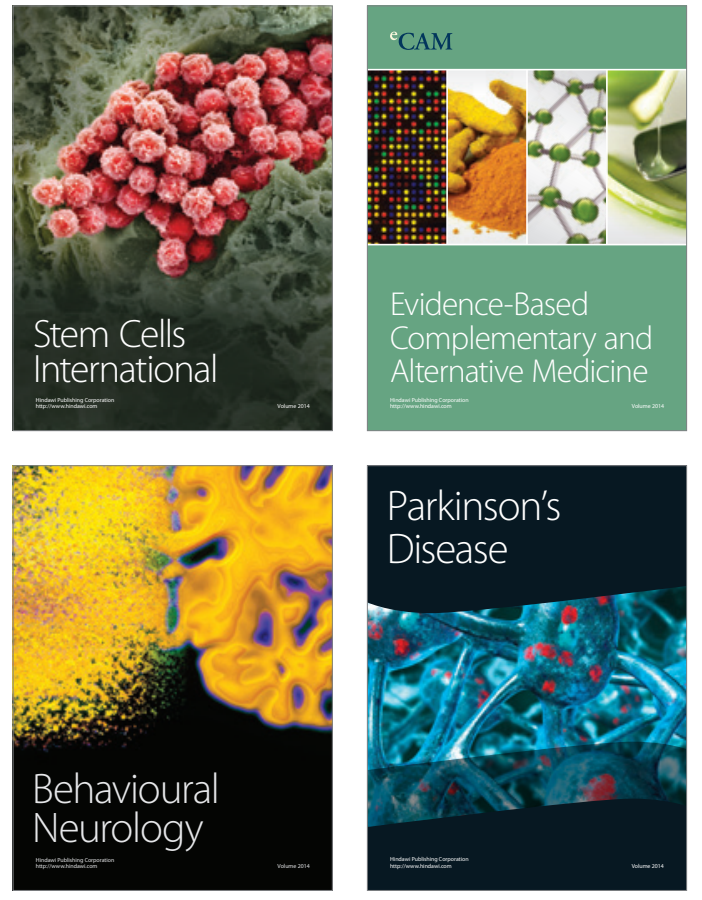
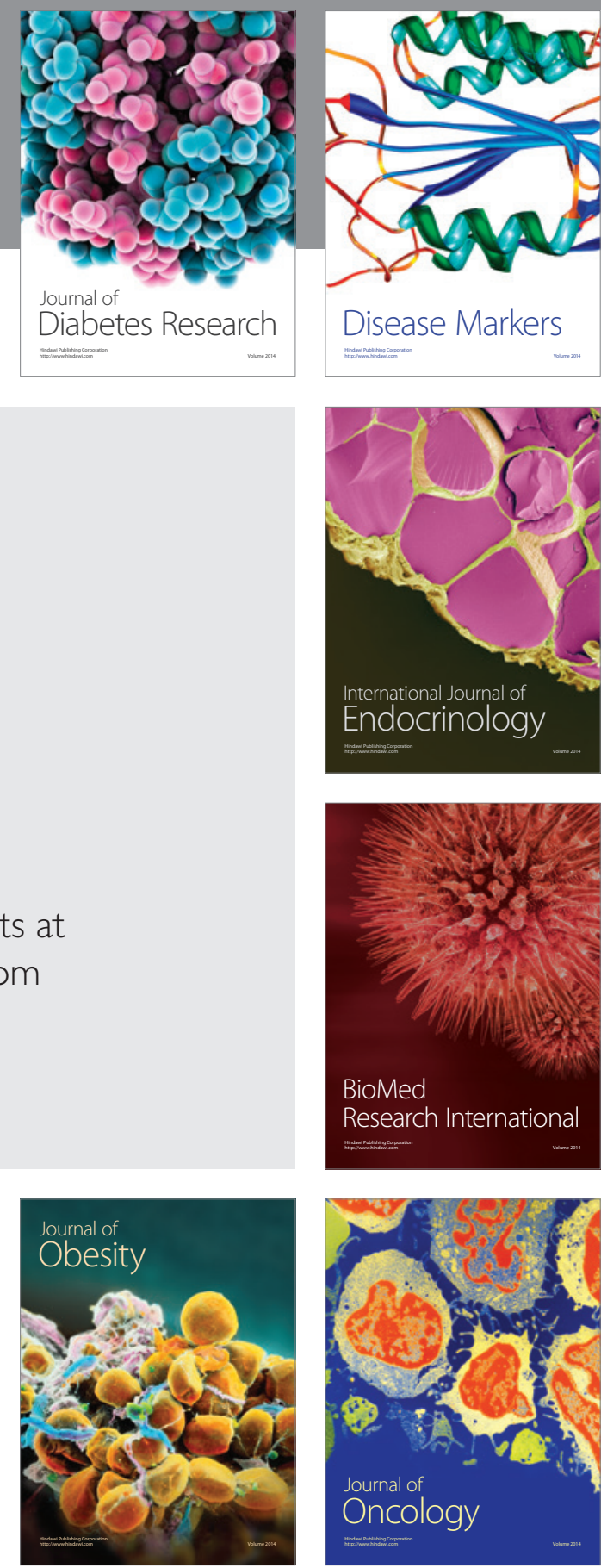

Disease Markers
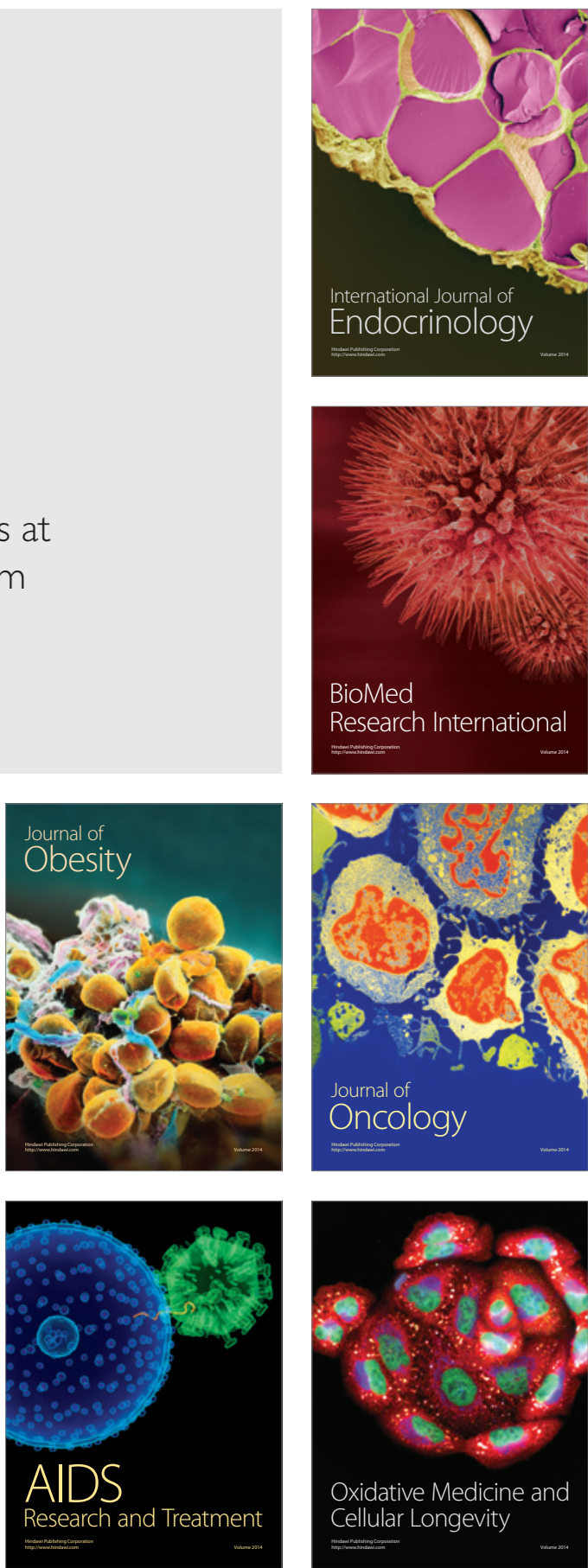